\title{
Pierre Auger Observatory: Status Report
}

\author{
M. A. L. de Oliveira \\ Depto. de Raios Cósmicos e Cronologia, Instituto de Física Gleb Wataghin \\ Universidade Estadual de Campinas, Cx.P. 6165, CEP 13084-971, Campinas, SP, Brasil \\ (Representing the Pierre Auger Collaboration)
}

Received on 8 December, 2003

\begin{abstract}
The Pierre Auger Observatory is a large international effort to provide measurements of extensive air showers (EAS) initiated in the upper atmosphere by cosmic rays with energies above $10^{18} \mathrm{eV}$, the Ultra High Energy Cosmic Rays (UHECR's). The explanations for the origin, acceleration processes and composition of the UHECR's face theoretical and experimental difficulties, due to the extremely low flux in this energy region. The full observatory will consist of a hybrid detector using an array of 1600 water Čerenkov tanks spread over an area of $3000 \mathrm{~km}^{2}$ overlooked by a set of 4 air fluorescence detectors per site. The southern site is being instrumented in Argentina and I will discuss its present status in this paper.
\end{abstract}

\section{Introduction}

The Pierre Auger Observatory [1] is nowadays the world largest array of detectors exploring the energy spectrum, arrival distributions and composition of cosmic rays above $10^{18} \mathrm{eV}$. The UHECR's are one of the most puzzling mysteries of nature that has appeared in the last times. They are subatomic particles going through the universe and reaching the Earth with macroscopic energy $\left(10^{20} \mathrm{eV} \sim 16 \mathrm{~J}\right)$. The first observation of cosmic rays with energies above $5 \times 10^{19} \mathrm{eV}$ was done in 1962 by Linsley [2] in the Volcano Ranch array. See the reference [3] for a very detailed overview of this subject. Since then, several events with energy above $10^{20} \mathrm{eV}$ have been observed [4] [5] [6]. However, the previous arrays, with collecting area $<100 \mathrm{~km}^{2}$, do not provide enough statistics, due to the extremely low flux ( 1 event century $\mathrm{H}^{-1} \mathrm{~km}^{-2}$ ) in this energy region [7] [8]. The explanation of these events also face theoretical difficulties, since it is believed to exist a cutoff in the spectrum of cosmic rays around $5 \times 10^{19} \mathrm{eV}$ due to the interaction with the microwave cosmic background photons, the GZK cutoff [9] [10]. Therefore, the scientific objectives of the observatory are to understand the nature, origin and propagation of UHECR's: how can these particles gain such tremendous energies? Are there point sources or are the arrival directions isotropic? Does the GZK cutoff exists or is there a continuing spectrum? Are the UHECR's accelerated or results of the decay of exotic particles? What are the primary masses? To answer these questions, the observatory will detect cosmic rays with an aperture greater than $7000 \mathrm{~km}^{2} \mathrm{sr}$ at $10^{19} \mathrm{eV}$ providing high statistics. It will fully cover the sky with uniform exposure and angular resolution of 1 degree.

The observatory is a hybrid giant detector using two different techniques (see Fig. 1): water Čerenkov tanks, the Surface Detector (SD), and fluorescence telescopes, the Fluorescence Detector (FD). The first technique consists of an array of 1600 tanks separated by $1.5 \mathrm{~km}$ spread over an area of $3000 \mathrm{~km}^{2}$. The UHECR's are so rare that even with such a large area, extrapolating the AGASA spectrum [11] below $10^{20} \mathrm{eV}$, we will have approximately 103 events per year. Some advantages of the Cerenkov tanks are their low cost and the fact that this kind of detector can operate continuously with any kind of weather. The ground array samples the lateral distribution of the shower generated by the primary UHECR's. The second technique consists of 24 telescopes overseeing the array of tanks. These telescopes observe the fluorescence light emitted by the air nitrogen molecules when they are excited by the particles of an EAS. They operate with clean weather in moonless nights. This condition corresponds to only $10 \%$ of the available time, but this disadvantage is compensated by the crucial information that the fluorescence detector gives to us: the longitudinal distribution profile of the EAS. This information is used in order to determine the energy, the arrival direction and to estimate the composition of the UHECR that originated the showers. Each one of the 24 telescopes is composed by a spherical mirror with $30^{\circ} \times 30^{\circ}$ field of view reflecting the fluorescence light on an array of 440 photomultiplier tubes (PMT's). The system adopts Schmidt optics [12] in order to reduce coma aberration. The basic setup is an aperture box with $110 \mathrm{~cm}$ of diaphragm radius with an external ring of corrector lenses and a band pass filter passing the $300 \mathrm{~nm}<\lambda<410 \mathrm{~nm}$ band, which contains the main emission peaks of the nitrogen fluorescence.

\section{The Surface Detector}

Water Čerenkov detectors has been selected as the technology for the ground array. In Fig. 2, we can see the detail of a unity of the SD. These are polyethylene resin tanks with capability of storage of $12000 \mathrm{l}$ of water $\left(10 \times 1.2 \mathrm{~m}^{3}\right)$. The stability of the water is critical for the successful long term operation, where the two sources of contamination, chemi- 


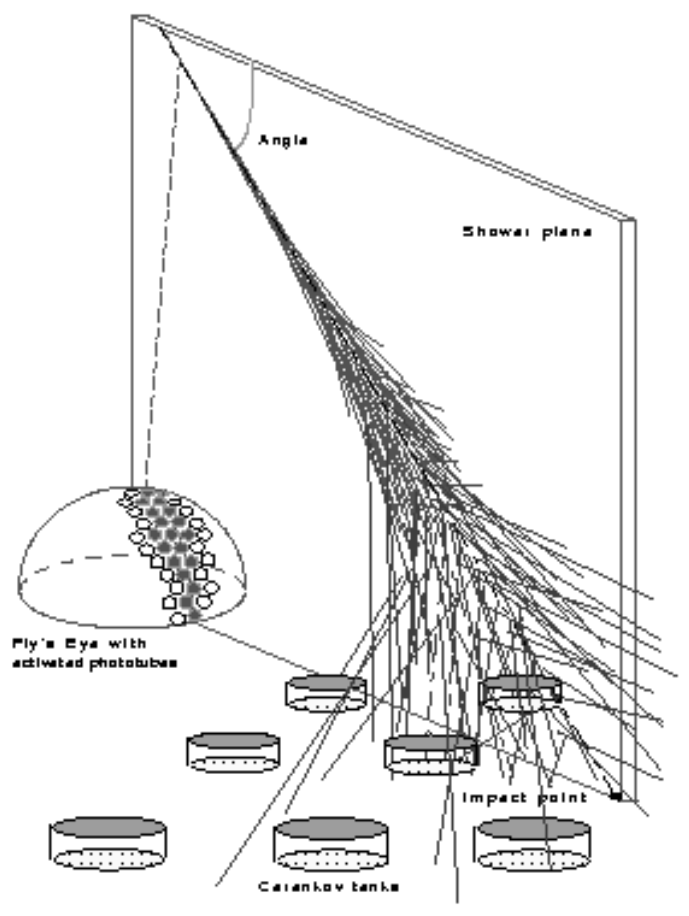

Figure 1. Illustration of the hybrid technique.

cal and biological activities, are controlled by separating the rusting component from the water by using a plastic barrier of Tyvek-polyethylene. Three PMT's Photonis XP1805 are positioned $1.2 \mathrm{~m}$ above the bottom water level looking downward. A very good proportionality between Čerenkov light and photoelectron yield is obtained for the PMT's placed at $120^{\circ}$ interval on a circle. Instead of connecting the detectors (which would be too costly), it has been decided to operate each detector in a stand-alone mode.

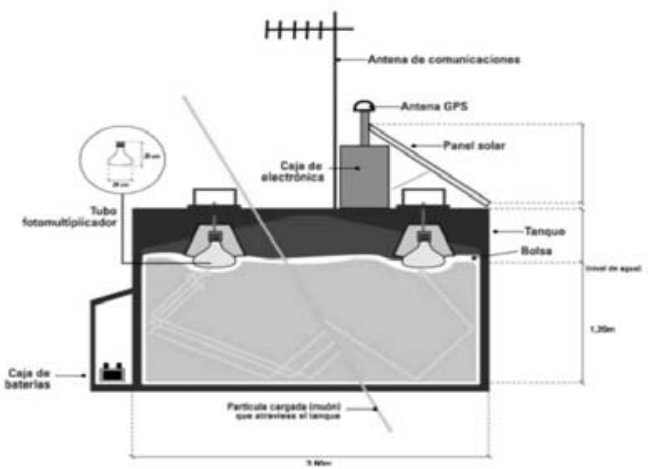

Figure 2. The water Čerenkov tank.

The power is obtained from solar panels while data are synchronized in time using GPS receivers. The trigger data is sent to the central station using a wireless local area network (LAN) radio link operating in $915 \mathrm{MHz}$. The power consumption of an SD station is below $10 \mathrm{~W}$.

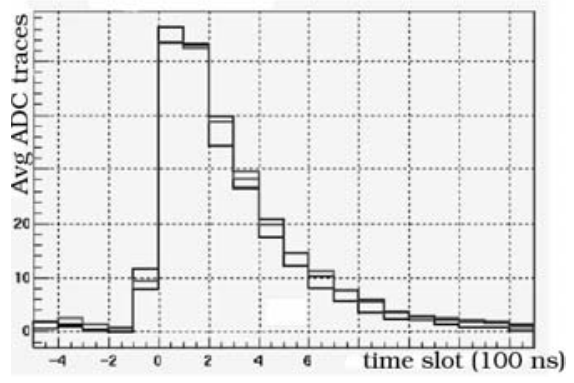

Figure 3. The three PMT's flash ADC traces for single muon signals.

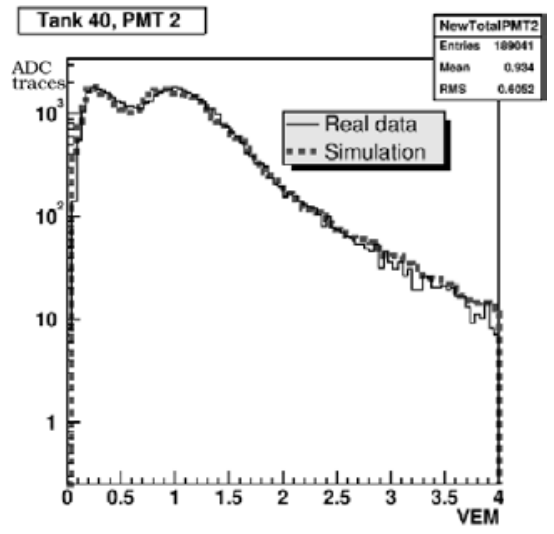

Figure 4. Signals from a auto trigger operation in units of VEM.

The signals from the PMT's are integrated in flash ADC electronics which digitally measures the signals every $100 \mathrm{~ns}$. Since there will be 1600 tanks allocated in the site operating for a long time, each of them with 3 PMT's, the monitoring of the signals is a considerable task. The detectors are monitored and calibrated by measuring the pulse height and time of single muons setting the high voltages to levels to maintain the tank trigger rate to a constant value. In Fig. 3, is shown the ADC traces of each PMT for a typical single muon signal in the tank. The gain is adjusted to keep the three readings comparable and then one can determine the vertical equivalent muon (VEM) value. In Fig. 4, one can see the signals given in units of VEM for a single trigger operation, as well as the simulation. The second bump is assumed for signals of muons passing through the entire tank depth, while the first one is accounted for electrons, gamma induced cascades and very inclined muons. The array trigger is formed in the Central Data Aquisition System (CDAS) system taking the coincidence of at least three detectors. The SD will sample the lateral distribution of particles and the time of arrival of the shower front. These measurements provide information to calculate the energy and the arrival direction of the shower primary. The response of the array is largely independent of the daylight luminosity and atmospheric conditions giving a duty cycle of $100 \%$ and an uniform coverage in right ascension over a day. It also has a good sensitivity to showers at large zenith angles [13]. 


\section{The Fluorescence Detector}

Around the perimeter of the SD there will be four stations of the FD (Los Leones, Cohiueco, Morados and Pocitos). Each station is divided in six bays with telescopes covering a field of view of $30^{\circ} \times 30^{\circ}$ (see Fig. 5). The telescope consists of a set of spherical mirrors of $340 \mathrm{~cm}$ of curvature radius, where the aperture is limited by a diaphragm of $220 \mathrm{~cm}$ of diameter put in the plane of the mirror curvature center (see Fig. 6). Half of the FD mirrors will be made from solid aluminum milled of a cast aluminum backing to the appropriate radius and protected by electromechanical indication. The other half will be made from high quality hexagonal glass segments, with an reflective aluminum layer deposited in vacuum and covered by a $\mathrm{SiO}_{2}$ layer. In front of the mirrors curvature center, acting as the window of the bay, there is a broadband pass MUG-6 filter glass (by Schott-Desag) $3.25 \mathrm{~mm}$ thick. It transmits light efficiently in the near UV wavelength region $(300 \mathrm{~nm}<\lambda<410 \mathrm{~nm}$ ), thus selecting the main emission peaks of the nitrogen fluorescence in the atmosphere, while attenuates visible light reducing the background noise. In order to correct the rays far apart from the main optical axis, a corrector lens ring of $25 \mathrm{~cm}$ of width is installed [14]. The idea of a corrector lens appeared as a way to increase the telescope area, increasing the spot luminosity, without increasing the spot size in excess. The shape of a ring has been chosen instead of a in filled lens to reduce the final cost and because the simulations showed that it was needed to correct only the rays beyond $85 \mathrm{~cm}$ from the main optical axis. The lens segments are made of BK7 borosilicate glasses and are ground and polished with an appropriate profile (given by simulation [15]). The resulting spot size is about $0.5^{\circ}$ in the focal surface, approximately one third of the pixel diameter. The light is captured at the focal surface by a camera of 440 PMT's Phillips XP3062 in an array of $20 \times 22$ tubes. To increase uniformity, in the space between the hexagonal tubes, is inserted light reflectors (the Merce$d e s$ ). The increase in the light capture efficiency can be seen in Fig. 7. Pixel trigger rates are kept close to $100 \mathrm{~Hz}$ and the candidates for cosmic ray events are selected by a powerful pattern recognition system which sends to CDAS an order to initiate a SD trigger. This procedure stamps a flag in hybrid events, which are very important for cross checking the reconstructions results of each technique. The telescopes track the paths of the charged particles along the shower axis, sampling its longitudinal development. This information is used to calculate the energy, the arrival direction and the mass composition of the shower primary. For the FD there is also the possibility of having stereo events, observed by at least two stations. The absolute calibration is achieved by a set of three procedures:

1. Pulse led lights into diffusers at three different positions of the telescope (piecewise calibration).

2. Pulse led into a diffuser drum with the same size of the aperture diaphragm, generating a plane wave which impinges on the mirror (end-to-end calibration).

3. Shoot laser in the atmosphere and recording the response to Rayleigh scattering (absolute calibration).
Recently, it has been reported [16] the calibration of 4.1 photons at the front of the aperture per FADC count. The uncertainty in this measurement was estimated to be $12 \%$. A Central Laser Facility (CLF) is operational since 2003 near the center of the SD array to provide regular laser shots.

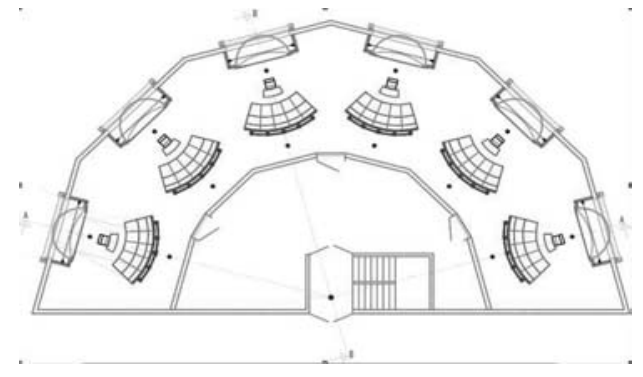

Figure 5. Upper view of a FD station.

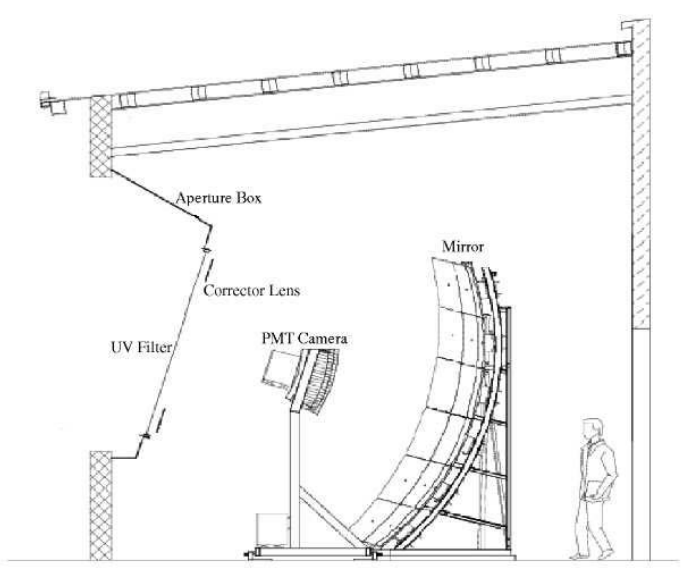

Figure 6. Schematic view of a FD telescope optics.

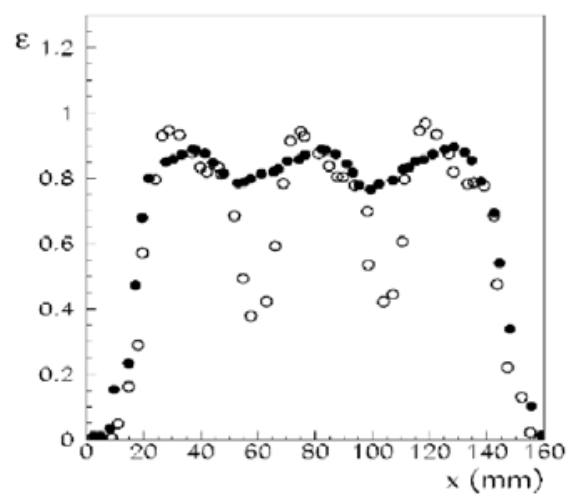

Figure 7. Efficiency in light collection without Mercedes (open circles) and with Mercedes (black circles). 


\subsection{The Atmosphere Monitoring}

The atmosphere conditions must be precisely monitored. The photons produced by the nitrogen fluorescence in shower axis must travel through the air to reach the telescopes. In their way, they will be scattered by air molecules (Rayleigh) and by aerosols (Mie) and be lost. In the counterpart, Cerenkov photons may be scattered toward the telescope. All these processes are dependent on the atmosphere conditions like density, temperature, pressure, humidity and dust concentration. The total light profile and the contributions due to direct and scattered Čerenkov radiation is plotted in Fig. 8. In order to accurately measure the number of particles in the shower axis, one has to discount the Čerenkov contributions. LIDAR systems have been used to monitor the atmospheric conditions [18]. Using a frequency tripled $\mathrm{Nd}$ :YAG laser able to transmit up 15 shots per second of $4 \mathrm{~ns}$ duration and output power of $\sim 6 \mathrm{~mJ}$, emitting in the wavelength of $355 \mathrm{~nm}$ within the fluorescence band. The receiver is made of a $90 \mathrm{~cm}$ parabolic mirror and $64.5 \mathrm{~cm}$ of focal length, the mirror is made of aluminum coated pyrex protected with $\mathrm{SiO}_{2}$ (formerly the EASTOP Čerenkov setup [17]). The backscattered light is detected by a Hammamatsu R7400 PMT with voltage set to $1000 \mathrm{~V}$ and a broad band UG-1 filter is used in order to reduce background. The distance between laser beam and mirror center is fixed to $1 \mathrm{~m}$ and the system is fully steerable with $0.1^{\circ}$ angular resolution. A recent extensive analysis of over 20 hours of atmospheric data taking $(\sim 1.9 G B)$ has been reported [19], concluding that the LIDAR station is able to produce stable data with quality and can probe the atmosphere up to $20 \mathrm{~km}$ altitude following reasonable signal to noise constraints.

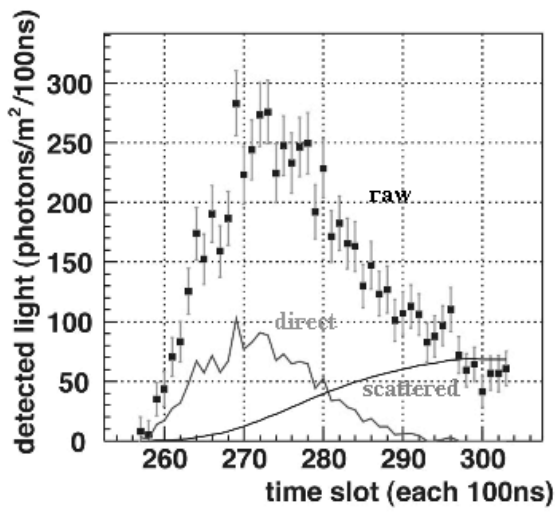

Figure 8. FD raw data and Čerenkov contaminations.

\section{The Engineering Array}

Before constructing the full size observatory, a smaller subset of it, the Engineering Array (EA) [20], has been built and operated to evaluate materials and methods of the proposed designs. For the SD, the EA permitted the testing of PMT's, electronics, cables, solar panels, batteries, liners and tank plastics and rotomoldings. Water production and quality as well as deployment strategies have been studied.
Softwares, triggers, monitoring packages, telecommunications and data acquisitions were tested. 40 SD units were deployed and 32 completely instrumented, covering an area of $46 \mathrm{~km}^{2}$. For the FD, the EA permitted the testing of mirrors, corrector rings, UV-pass filters, camera of PMT's, aperture boxes, shutters and slow control. Also, it was possible to test triggers, acquisition systems and softwares, calibration procedures and communications. Two FD units were instrumented at Los Leones building (10 $\mathrm{km}$ to the south of the surface EA center, see Fig. 9). Near the center of EA array an extra detector was placed $11 \mathrm{~m}$ from an existing one, forming a pair (Carmen and Miranda) used to measure timings and densities from essentially the same distance from the shower core. Another two detectors were placed at $860 \mathrm{~m}$ from the nearest neighbors of the Carmen-Miranda pair allowing lower energies showers triggering. The EA was operational from the beginning of 2003 to mid 2002, the SD takes data continuously since then, while the FD was switched off to be upgraded with the final hardwares. The EA phase was greatly successful, some improvements have been made to the original proposals, but essentially the experiment and the individual components worked satisfactorily.

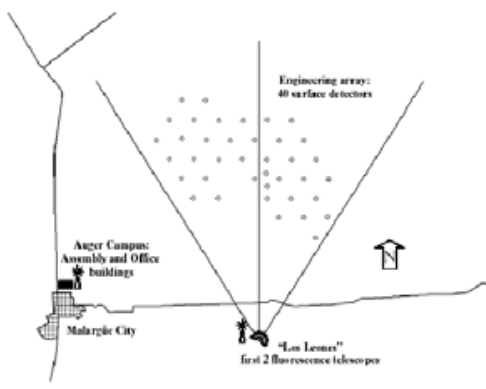

Figure 9. The Engineering Array map.

\section{Event Reconstructions}

Up till now more than 600000 SD triggers have been recorded. A typical event above $10^{19} \mathrm{eV}$ is shown in Fig. 10. The shower geometry is given by time-of-flight technique. This event has a zenith angle of $48.8^{\circ}$ and azimuth angle of $-167.1^{\circ}$. In the upper left of the figure, one can see the list of triggered stations with respective VEM signals, these stations are represented in the site map (lower left) by the green circles, whose radii are proportional to their signals, and the red arrow represents the projection of the reconstructed shower axis and the impact point of the shower core. In the upper right, one can see the fit of the lateral distribution function through the stations densities, this fit allows the calculation of the signal far from the core, the $S(1000)$, where the fluctuations of the density of shower particles are quite small and hence the signal depends only on primary energy. This signal is used as energy estimator through a relation like: $\quad E_{0}=\alpha S(1000)^{\beta}$

where the parameters $\alpha$ and $\beta$ are calculated by simulation. The highest multiplicity event recorded during the EA phase is shown in Fig. 11. It fired 11 stations, the primary energy is calculated around $5 \times 10^{19} \mathrm{eV}$ and the large zenith angle $\left(82^{\circ}\right)$ is the cause of its high multiplicity. Recently, it has been reported [21] an even higher multiplicity event: 15fold event with energy above $5 \times 10^{19} \mathrm{eV}$ and zenith angle of $\sim 40^{\circ}$. 


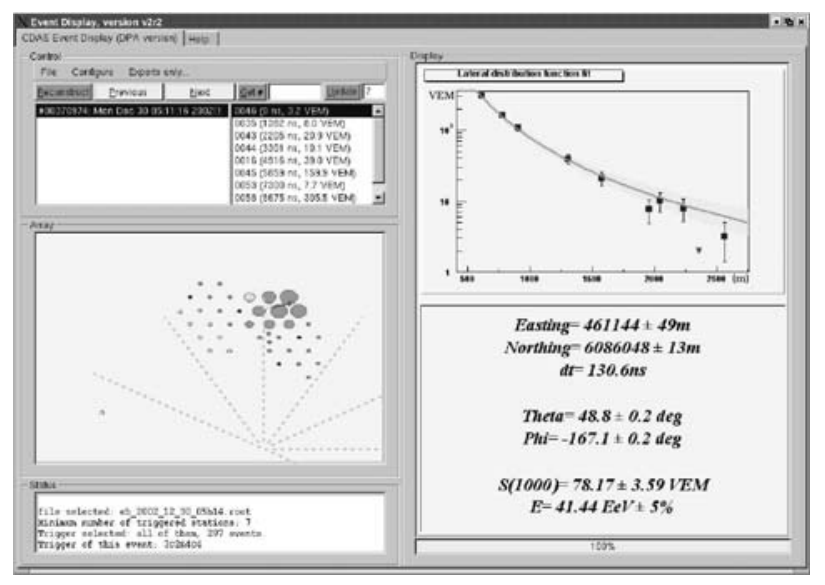

Figure 10. The display of SD event 370974.

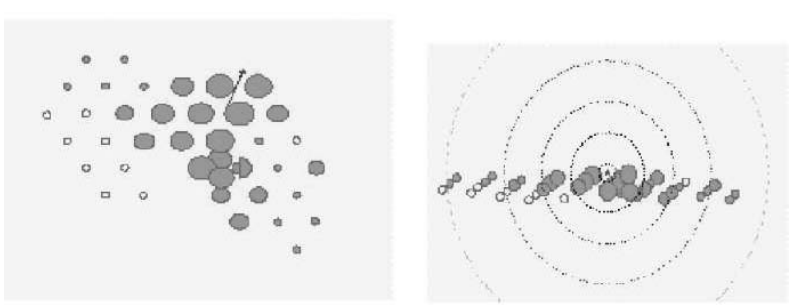

Figure 11. The highest multiplicity event for the EA phase: event 204272

The events recorded by the FD are reconstructed in following way (see Fig. 12):

1. The fluorescence track projection into the PMT camera (see Fig. 13) defines the Shower Detector Plane (SDP).

2. From the angular velocity through the PMT camera, one deduces the shower geometry $\left(R_{p}\right.$ and $\left.\psi\right)$, since the track travels at constant light speed.

3. From the pulse height distribution is calculated the longitudinal profile of particles at the shower axis (see Fig. 14), taking into account the atmosphere lost and Čerenkov contaminations, as discussed before.

One example of a reconstructed event of energy $10^{19} \mathrm{eV}$ is shown in Fig. 14, the maximum of the shower development is at the depth of $750 \mathrm{~g} / \mathrm{cm}^{2}$. The resolution on the determination of the shower maximum is important for the knowledge of the primary composition, since showers from heavier nuclei develop higher in the atmosphere. The energy of the particle which initiated the cascade is obtained from the track length integral of the longitudinal distribution:

$$
E_{e m}=\frac{E_{c}}{\lambda_{r}} \int N e(X) d X
$$

where $E_{e m}$ is the energy dissipated in the atmosphere by the electromagnetic component, $E_{c} / \lambda_{r}$ is the ratio of the critical energy to the radiation length and $N(X)$ is the observed number of electrons as a function of the atmospheric depth. An upward correction of $10 \%$ must be made in order to account for the missing energy of the muon, neutrino and hadronic components. A very detailed study of the missing energy was given by [22].

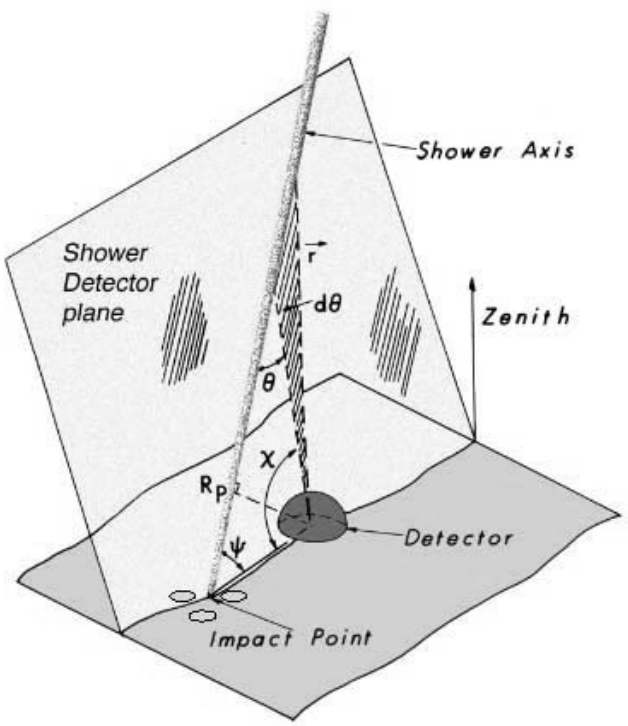

Figure 12. FD geometry reconstruction.

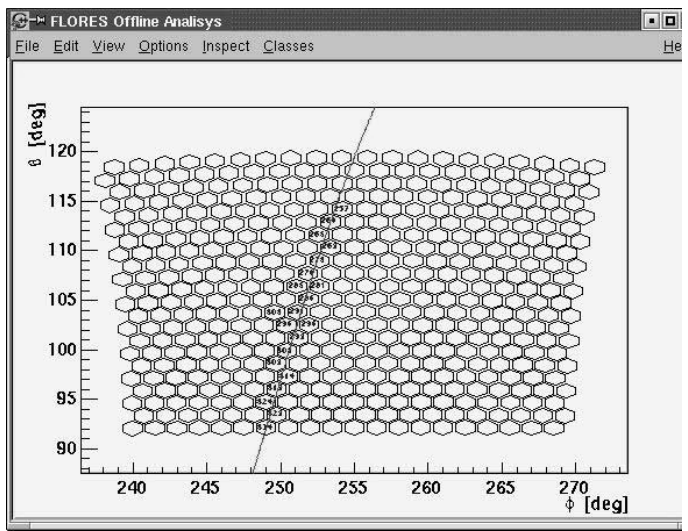

Figure 13 . The SDP seen by a PMT camera.

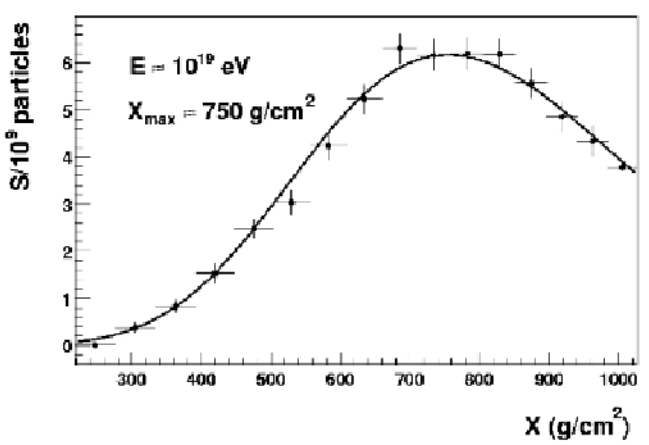

Figure 14. The fit of a Gaisser-Hillas function in the longitudinal profile. 
TABLE I. Comparison between surface only and hybrid accuracies [1], pp. 144.

\begin{tabular}{|c||c|c|c|c|}
\hline Energy & $10^{19} \mathrm{eV}$ & $10^{19} \mathrm{eV}$ & $10^{20} \mathrm{eV}$ & $10^{20} \mathrm{eV}$ \\
\hline Method & Surface & Hybrid & Surface & Hybrid \\
\hline \hline$\Delta \theta$ & $2.00^{\circ}$ & $0.35^{\circ}$ & $1.00^{\circ}$ & $0.36^{\circ}$ \\
\hline$\Delta$ core & $80 \mathrm{~m}$ & $29 \mathrm{~m}$ & $40 \mathrm{~m}$ & $29 \mathrm{~m}$ \\
\hline$\Delta E / E$ & $18 \%$ & $4.2 \%$ & $7 \%$ & $2.5 \%$ \\
\hline$\Delta X_{\max }$ & & $17 \mathrm{~g} / \mathrm{cm}^{2}$ & & $15 \mathrm{~g} / \mathrm{cm}^{2}$ \\
\hline
\end{tabular}

An important aspect of the observatory is the possibility of observing hybrid events, allowing cross-calibration of the $\mathrm{SD}$ and FD reconstructions. The energy reconstruction in $\mathrm{SD}$, for instance, are known to be model dependent, while in FD not. Thus, hybrid events allow to test models at Auger energies. However, many hybrid events are below the array threshold having less than three triggered stations. This fact difficulty's the direct comparison of both reconstructions, but may help in FD geometry reconstruction. Taking the arrival time at one tank, for example, one can constrain the core position in some tenths of meters [23]. Then, the information that comes from the particle density can be used to check if the model used to generate the lateral distribution function is well behaved for the SD. In table I is summarized the reconstruction errors for various quantities in two given energy levels for the surface array only and the improvement expected for hybrid events.

Besides the possibility of hybrid reconstruction, for the FD there is also the possibility of stereo events, i.e., events seen by (at least) two FD eyes. In these events, the calculation of the shower geometry is done by the intersection of two SDP's. A detailed study of the trigger performances for stereo and hybrid configurations using four telescopes and the present SD array has been made [24]. Using 1500 simulations [25] of $10^{19} \mathrm{eV}$ showers spread over an area $1,257 \mathrm{~km}^{2}$, it has been found an increasing in the trigger rate of $86 \%$ for stereo events (with two telescopes at Los Leones and two telescopes at Cohiueco), compared whith another configuration (three telescopes at Los Leones and one telescope at Cohiueco). This last configuration increased the hybrid trigger rate in $30 \%$.

Several events have been reported lately [26]: a very interesting event can be seen in Fig. 15. With energy of $2.54 \times 10^{19} \mathrm{eV}$ and zenith angle of $56.5^{\circ}$, this event fired 13 SD stations and the FD. In the event map, one can see the direction of the SDP plane given by the FD (symbolized by the dashed red line) which lies right on the SD reconstructed impact point on the ground. Another interesting event is shown in Figs. 16 and 17, this is a hybrid event as well, but in particular, it is one which could be seem by 2 adjacent telescopes, notice its track finishing at one mirror and stating exactly in the same elevation in the other mirror.

The observatory entered the Pre-Production phase: an array of 100 tanks and 2 FD eyes with 6 telescopes will become operational by the beginning of 2004. Since Friday October 3rd, after the SD Station Stroustrup woke up to CDAS and joined the Array as the 100th running station, the Auger is the largest running array in the world. The Southern Pierre Auger Observatory completion is expected for the end of 2005 .

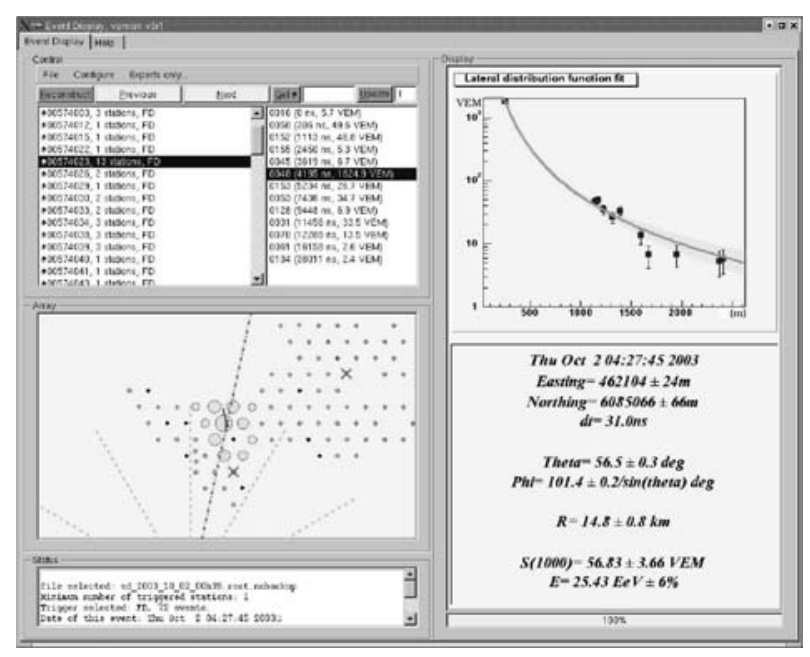

Figure 15. The SD event 574023.

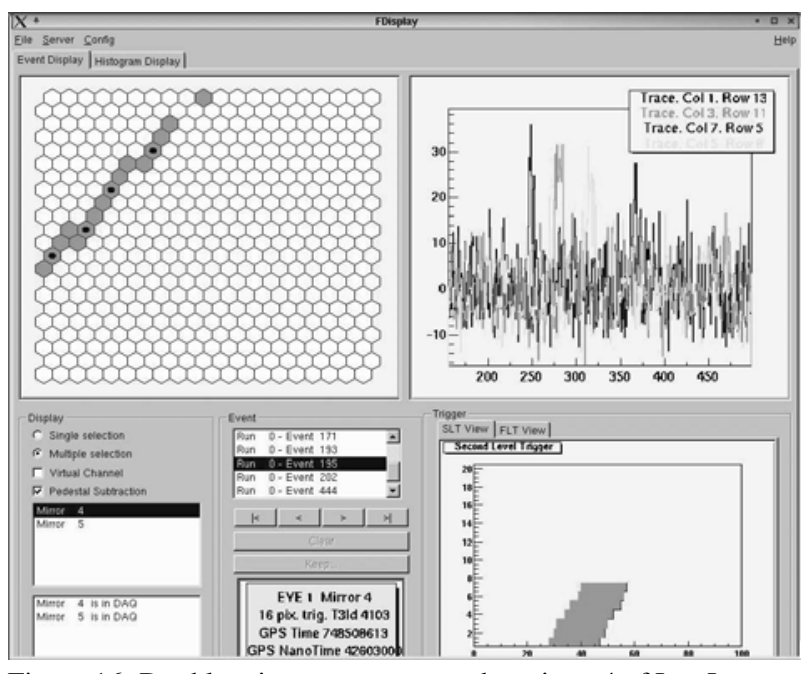

Figure 16. Double mirror event as seen by mirror 4 of Los Leones.

\section{Conclusion}

The Pierre Auger Observatory has recently ended the Engineering Array phase with great success. Several concepts, procedures and materials were tested and upgraded to the final designs. The prototype of the FD has ran and collected 


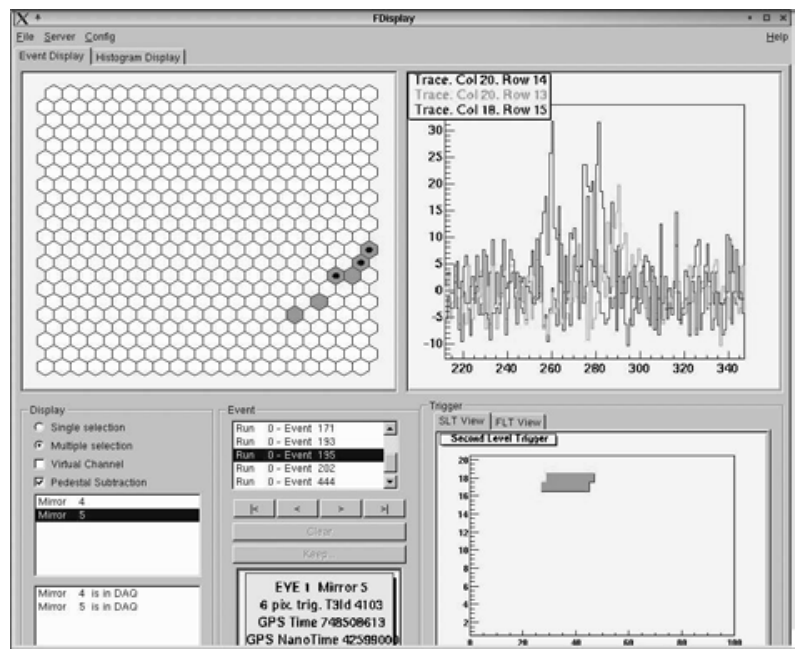

Figure 17. Double mirror event as seen by mirror 5 of Los Leones.

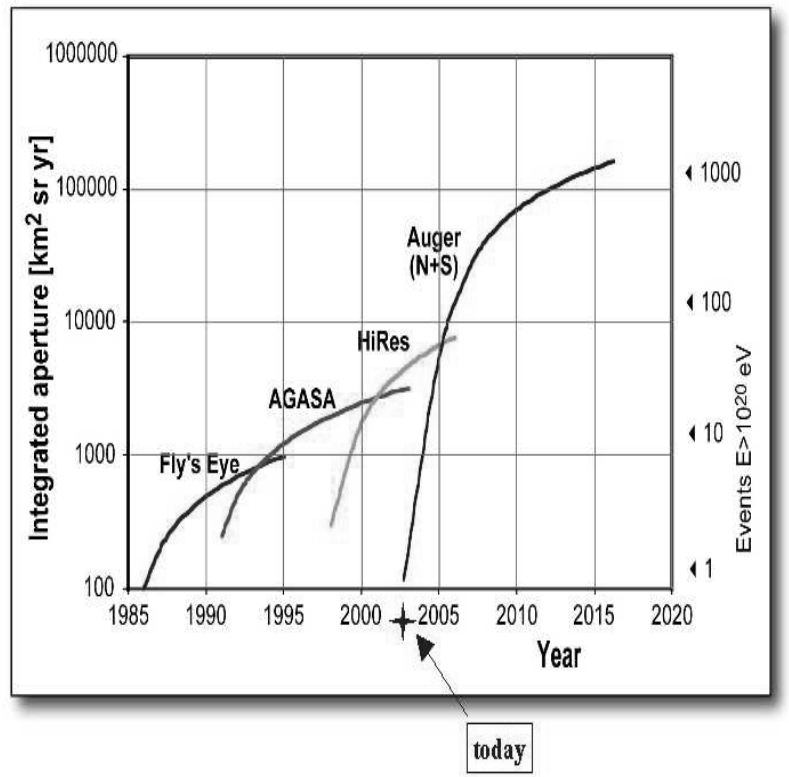

Figure 18. Integrated aperture of several experiments along the years [27].

events as expected and then dismantled for the assembling of of the final systems, while the SD is continuously growing and running. The Pre-Production phase has begun and by 2004 is expected 300 SD units and 2 fully installed FD telescopes. The Southern site will be finished by the end of 2005 and the construction of the Northern site will begin by 2006. The completion of the full sky coverage is expected by the year 2010 and afterwards. In the Fig. 18, we can see the increase of the integrated aperture as a function of time for several cosmic ray experiments in comparison with the Pierre Auger Observatory, in the right column is plot the expected number of events per year above $10^{20} \mathrm{eV}$. A flux grater than 1000 per year will be available sometime after 2012, which is enough statistics to provide some answers to the puzzle of the UHECR's.

\section{Acknowledgments}

The author would like to thank to the organizing committee of the XXIV Brazilian National Meeting on Particles and Fields and express his gratitude to Prof. Carlos Escobar from Unicamp for his support during all these years. This work is supported by Fapesp and CNPq (Brazil).

\section{References}

[1] Pierre Auger Design Report, Fermilab (1997), http://www.auger.org/admin/DesignReport/index.html

[2] J. Linsley, Phys. Rev. Lett., 34, 146 (1963).

[3] M. Nagano and A. A. Watson, Rev. of Modern Phys., 72, 689 (2000).

[4] D. J. Bird et al, Astrophys. J., 441, 144 (1995).

[5] N. Hayashida et al, Phys. Rev. Lett., 73, 3491 (1994).

[6] M. A. Lawrence, R. J. O. Reid, and A. A. Watson, J. Phys., G17, 733 (1991).

[7] M. Takeda et al, Astro/ph-0209422, Nov. (2002).

[8] D. J. Bird et al, Phys. Rev. Lett., 71, 3401 (1993).

[9] K. Greisen, Phys. Rev. Letters, 16, 748 (1966).

[10] G. T. Zatsepin and V. A. Kuz'min, JETP Lett., 4, 78 (1966).

[11] S. Yoshida et al, Astroparticle Phys., 3, 105 (1995).

[12] M. Born and E. Wolf, Principles of Optics, Cambridge University Press (1980).

[13] J. Knapp, Proc. of 2nd Int. Workshop on EHE Cosm. Rays, Nov. 5 (2002), Tokyo, Japan.

[14] M. A. L. de Oliveira, H. C. Reis, R. Sato, and V. de Souza, Manufacturing the Schmidt Corrector Lens for the Pierre Auger Observatory, accepted for publication in Nuclear Instruments and Methods $\mathbf{A}$.

[15] R. Sato, J.A. Bellido C., and H.C. Reis, Improving the $S / N$ Ratio for the Auger Fluorescence Detector, GAP Note 2000009, http://www.auger.org/admin-cgi-bin/woda/gap_notes.pl

[16] M. D. Roberts, P. Sommers, and B. Fick, Absolute calibration of the Auger fluorescence detectors using atmosphere laser beams, GAP Note 2003-010, http://www.auger.org/admincgi-bin/woda/gap_notes.pl

[17] M. Aglietta et al., Astrop. Phys., 6, 143 (1997).

[18] A. Filipic, M. Horvat, D. Verberic, D. Zavrtanik, and M. Zavrtanik, Analysis of Lidar Measurements at the Pierre Auger Observatory, GAP Note 2003-002,

http://www.auger.org/admin-cgi-bin/woda/gap_notes.pl

[19] R. Biral, Analysis of the 2002 Malargue Lidar data through Fernold's method, GAP Note 2003-008, http://www.auger.org/admin-cgi-bin/woda/gap_notes.pl 
[20] J. Abraham et al., Properties and Performance of the Prototype Instrument for the Pierre Auger Observatory, submitted to Nucl. Instr. and Meth. A(2003).

[21] X. Bertou, Communication at the Auger e-mail list: auger_collaboration@fnal.gov, Dec.1st (2003).

[22] H. M. J. Barbosa, F. Catalani, C. Dobrigkeit, and J. A. Chinellato, Study of the Energy Deposit in the Atmosphere by UHECR: The Missing Energy, GAP Note 2003-069, http://www.auger.org/admin-cgi-bin/woda/gap_notes.pl

[23] V. de Souza, C. Dobrigkeit, and B. Dawson, A hybrid reconstruction program and the hybrid resolution, GAP Note 2003069, http://www.auger.org/admin-cgi-bin/woda/gap_notes.pl
[24] Luis Prado Jr, Study of Hybrid and Stereo Trigger Performance for Different Detector Configurations with 4 Telescopes and the Pre-production Array, GAP Note 2003-044, http://www.auger.org/admin-cgi-bin/woda/gap_notes.pl

[25] Luis Prado Jr, FDSim User's Manual - PreProd v2ro, GAP Note 2003-084,

http://www.auger.org/admin-cgi-bin/woda/gap_notes.pl

[26] A. Letessier-Selvon, Communication at the Auger e-mail list: auger_collaboration@fnal.gov, Oct. 2nd (2003).

[27] H. Bluemer, Proc. 28th Int. Cosmic Ray Conf., TsukubaJapan, HE, 369-372 (2003). 